\title{
Mosco convergence results for common fixed point problems and generalized equilibrium problems in Banach spaces
}

\author{
Muhammad Aqeel Ahmad Khan ${ }^{1 *}$, Hafiz Fukhar-ud-din ${ }^{1,2}$ and Abdul Rahim Khan²
}

Dedicated to Professor Wataru Takahashi on the occasion of his 70th birthday

*Correspondence:

itsakb@hotmail.com

${ }^{1}$ Department of Mathematics, The

Islamia University of Bahawalpur,

Bahawalpur, 63100, Pakistan

Full list of author information is

available at the end of the article

\begin{abstract}
In this paper, we propose and analyze an explicit type algorithm for finding a common element of the set of solutions of a finite family of generalized equilibrium problems and the set of common fixed points of two countable families of total quasi- $\varphi$-asymptotically nonexpansive mappings in a Banach space $E$. As an application of our result, we suggest a framework for finding a common solution of a finite family of generalized equilibrium problems and common zeros of two finite families of maximal monotone operators on $E$.

MSC: 47H05; 47H10; 47H15; 47J25; 49M05
\end{abstract}

Keywords: total quasi- $\varphi$-asymptotically nonexpansive mapping; asymptotic fixed point; equilibrium problem; variational inequality problem; maximal monotone operator; Mosco convergence

\section{Introduction and preliminaries}

Let $E$ be a real Banach space with the norm $\|\cdot\|$ and $E^{*}$ be its dual. Let $C$ be a nonempty subset of $E$ and $T: C \rightarrow C$ be a mapping. We denote by $F(T)=\{x \in C: x=T x\}$ the set of fixed points of $T$. We symbolize weak convergence and strong convergence of a sequence $\left\{x_{n}\right\}$ in $E$ as $x_{n} \rightarrow x$ and $x_{n} \rightarrow x$, respectively. Let $f: C \times C \rightarrow \mathbb{R}$ (the set of reals) be a bifunction and $A: C \rightarrow E^{*}$ be a nonlinear mapping. A generalized equilibrium problem is to find the set

$$
G E P(f)=\{x \in C: f(x, y)+\langle A x, y-x\rangle \geq 0 \text { for all } y \in C\},
$$

where $\langle\cdot, \cdot\rangle$ stands for the duality product.

Note that:

(i) if $A \equiv 0$, then problem (1.1) reduces to the following equilibrium problem $E P(f)$ :

$$
\text { find } x \in C \text { such that } f(x, y) \geq 0 \text { for all } y \in C \text {; }
$$

(ii) if $f \equiv 0$, then problem (1.1) reduces to the classical variational inequality problem $V I(C, A)$ :

find $x \in C$ such that $\langle A x, y-x\rangle \geq 0$ for all $y \in C$. 
The equilibrium problem provides a unified approach to finding a solution of a large number of problems arising in physics, optimization, economics and fixed point problems [1]. Moreover, the generalized equilibrium problem addresses monotone inclusion problems, variational inequality problems, minimization problems and vector equilibrium problem [2-4]. Since an algorithmic construction plays a key role in solving nonlinear equations in various fields of investigation, numerous implicit and explicit algorithms have been developed for the approximate solution of nonlinear equations as well as for the approximation of fixed points of various mappings [5-7].

Recall that a Banach space $E$ is said to be:

(i) strictly convex if for all $x, y \in S_{E}:=\{z \in E:\|z\|=1\}$ with $x \neq y$, we have $\|x+y\|<2$;

(ii) uniformly convex if for any $\epsilon \in(0,2]$, there exists $\delta>0$ such that

$$
x, y \in S_{E}, \quad\|x-y\| \geq \epsilon \quad \Longrightarrow \quad\left\|\frac{x+y}{2}\right\| \leq 1-\delta .
$$

A Banach space $E$ is said to have the Kadec-Klee property if for any sequence $\left\{x_{n}\right\}$ in $E$ with $x_{n} \rightarrow x_{0} \in E$ and $\lim _{n \rightarrow \infty}\left\|x_{n}\right\|=\left\|x_{0}\right\|$, we have $x_{n} \rightarrow x_{0}$. Note that every uniformly convex Banach space $E$ is strictly convex and enjoys the Kadec-Klee property but the converse is not true.

Furthermore, define $h: S_{E} \times S_{E} \times \mathbb{R} \backslash\{0\} \rightarrow \mathbb{R}$ by

$$
h(x, y, t)=\frac{\|x+t y\|-\|x\|}{t}
$$

for $x, y \in S_{E}$ and $t \in \mathbb{R} \backslash\{0\}$. The norm of $E$ is said to be Gâteaux differentiable if $\lim _{t \rightarrow 0} h(x, y, t)$ exists for each $x, y \in S_{E}$ and in this case $E$ is smooth.

The normalized duality mapping $J: E \rightarrow E^{*}$ is defined by

$$
J x=\left\{x^{*} \in E^{*}:\|x\|^{2}=\left\langle x, x^{*}\right\rangle=\left\|x^{*}\right\|^{2}\right\} \quad(x \in E) .
$$

It is remarked that the set-valued mapping $J$ is nonempty, closed and convex in a real Banach space whereas $J$ is single-valued in a reflexive, strictly convex and smooth Banach space. Furthermore, $J^{-1}: E^{*} \rightarrow E$, the inverse of the normalized duality mapping $J$, is also a duality mapping in a uniformly convex and smooth Banach space. Both $J$ and $J^{-1}$ are uniformly norm-to-norm continuous on each bounded subset of $E$ and $E^{*}$, respectively. If $E$ is reflexive and strictly convex, then $J^{-1}$ is norm-to-weak continuous. For more details, see $[8,9]$.

The Lyapunov functional $\varphi: E \times E \rightarrow \mathbb{R}$ is defined by

$$
\varphi(x, y)=\|x\|^{2}-2\langle x, J y\rangle+\|y\|^{2} \quad \text { for all } x, y \in E .
$$

It is obvious from the definition of $\varphi$ that $\varphi(x, y) \geq 0$ for all $x, y \in E$. In a real Hilbert space, $\varphi(x, y)=\|x-y\|^{2}$ for all $x, y \in E$. For details, see $[5,10]$.

Let $E$ be a reflexive, strictly convex and smooth Banach space, and let $C$ be a nonempty, closed and convex subset of $E$. Then, for arbitrarily fixed $x \in E$, there exists a unique point $y_{x} \in C$ such that $\varphi\left(x, y_{x}\right)=\min _{y \in C} \varphi(x, y)$. Following the notation of [5], we let $\Pi_{C}(x)=y_{x}$ and call $\Pi_{C}$ a generalized projection onto $C$. Note that the generalized projection operator coincides with the metric projection in a Hilbert space. 
A point $x \in C$ is said to be an asymptotic fixed point [11] of $T: C \rightarrow C$ if there exists a sequence $\left\{x_{n}\right\} \subset C$ such that $x_{n} \rightarrow x$ and $\lim _{n \rightarrow \infty}\left\|x_{n}-T x_{n}\right\|=0$. The set of all asymptotic fixed points of $T$ is denoted by $\widehat{F}(T)$.

Recall that a mapping $T: C \rightarrow C$ is:

(i) nonexpansive if $\|T x-T y\| \leq\|x-y\|$ for all $x, y \in C$;

(ii) relatively nonexpansive if $\widehat{F}(T)=F(T) \neq \emptyset$ and $\varphi(p, T x) \leq \varphi(p, x)$ for all $p \in F(T)$ and $x \in C$;

(iii) quasi- $\varphi$-nonexpansive if $F(T) \neq \emptyset$ and $\varphi(p, T x) \leq \varphi(p, x)$ for all $p \in F(T)$ and $x \in C$;

(iv) quasi- $\varphi$-asymptotically nonexpansive if there exists a real sequence $\left\{k_{n}\right\}$ with $k_{n} \geq 1 ; k_{n} \stackrel{n \rightarrow \infty}{\rightarrow} 1$ and $F(T) \neq \emptyset$ such that $\varphi\left(p, T^{n} x\right) \leq k_{n} \varphi(p, x)$ for all $n \geq 1, p \in F(T)$ and $x \in C$;

(v) total quasi- $\varphi$-asymptotically nonexpansive if there exist nonnegative real sequences $\left\{k_{n}\right\}$ and $\left\{\eta_{n}\right\}$ with $k_{n} \stackrel{n \rightarrow \infty}{\rightarrow} 0, \eta_{n} \stackrel{n \rightarrow \infty}{\rightarrow} 0$ and $F(T) \neq \emptyset$ such that

$$
\varphi\left(p, T^{n} x\right) \leq \varphi(p, x)+k_{n} \xi(\varphi(p, x))+\eta_{n} \quad \text { for all } n \geq 1, p \in F(T) \text { and } x \in C,
$$

where $\xi: \mathbb{R}^{+} \rightarrow \mathbb{R}^{+}$is a strictly increasing continuous function with $\xi(0)=0$.

It is worth mentioning that the class of total quasi- $\varphi$-asymptotically nonexpansive mappings properly contains the mappings defined in (i)-(iv), but the converse is not true.

A mapping $T: C \rightarrow C$ is said to be uniformly $L$-Lipschitzian if

$$
\left\|T^{n} x-T^{n} y\right\| \leq L\|x-y\| \quad \text { for some } L>0 \text { and } x, y \in C .
$$

Recently, numerous attempts have been made to guarantee strong convergence through explicit and implicit algorithms for finding a common solution of the set of fixed points of (relatively nonexpansive, quasi- $\varphi$-nonexpansive, quasi- $\varphi$-asymptotically nonexpansive, total quasi- $\varphi$-asymptotically nonexpansive) mappings and the set of solutions of equilibrium problems; see [12-21] and the references cited therein.

In 2008, Takahashi and Zembayashi [21] introduced an explicit algorithm based on the shrinking projection method for finding a common solution of the set of fixed points of a relatively nonexpansive mapping $T$ and the set of solutions of an equilibrium problem. Their algorithm reads as follows:

$$
\left\{\begin{array}{l}
x_{0}=x \in C=C_{0}, \\
y_{n}=J^{-1}\left(\alpha_{n} J x_{n}+\left(1-\alpha_{n}\right) J T x_{n}\right), \\
u_{n} \in C \text { such that } f\left(u_{n}, y\right)+\frac{1}{r_{n}}\left\langle y-u_{n}, J u_{n}-J y_{n}\right\rangle \geq 0 \quad \text { for all } y \in C, \\
C_{n+1}=\left\{z \in C_{n}: \varphi\left(z, u_{n}\right) \leq \varphi\left(z, x_{n}\right)\right\}, \\
x_{n+1}=\Pi_{C_{n+1}} x_{0}, \quad n \geq 0,
\end{array}\right.
$$

where $J$ is the duality mapping on $E$ and $\Pi_{C}$ is the generalized projection from $E$ onto $C$. They proved that the sequence $\left\{x_{n}\right\}$ generated by $(1.2)$ converges strongly to $\Pi_{F(T) \cap E P(f)} x_{0}$ under some appropriate conditions.

In 2010, Chang et al. [12] proved a strong convergence theorem for finding a common element of the set of solutions for generalized equilibrium problem (1.1) and the set of common fixed points for a pair of relatively nonexpansive mappings in Banach spaces. 
Their algorithm reads as follows:

$$
\left\{\begin{array}{l}
x_{0} \in C \text { chosen arbitrarily, } \\
z_{n}=J^{-1}\left(\alpha_{n} J x_{n}+\left(1-\alpha_{n}\right) J T x_{n}\right), \\
y_{n}=J^{-1}\left(\beta_{n} J x_{n}+\left(1-\beta_{n}\right) J S z_{n}\right), \\
u_{n} \in C \text { such that } f\left(u_{n}, y\right)+\left\langle A u_{n}, y-u_{n}\right\rangle+\frac{1}{r_{n}}\left\langle y-u_{n}, J u_{n}-J y_{n}\right\rangle \geq 0 \\
\quad \text { for all } y \in C, \\
H_{n}=\left\{z \in C: \varphi\left(z, u_{n}\right) \leq \beta_{n} \varphi\left(z, x_{n}\right)+\left(1-\beta_{n}\right) \varphi\left(z, z_{n}\right) \leq \varphi\left(z, x_{n}\right)\right\}, \\
W_{n}=\left\{z \in C:\left\langle x_{n}-z, J x_{0}-J x_{n}\right\rangle \geq 0\right\}, \\
x_{n+1}=\Pi_{H_{n} \cap W_{n} x_{0}, \quad n \geq 0,}
\end{array}\right.
$$

where $J, T, S$ and $\Pi_{C}$ are as in (1.2). The authors showed that the sequence $\left\{x_{n}\right\}$ generated by (1.3) converges strongly to $\prod_{F(S) \cap F(T) \cap G E P(f)} x_{0}$ under some appropriate conditions.

In 2009, Wattanawitton and Kumam [22] approximated a common solution for a pair of relatively quasi-nonexpansive mappings and an equilibrium problem. Recently, Qin et al. [18] established strong convergence results for a pair of asymptotically quasi- $\varphi$ nonexpansive mappings in a Banach space. It is worth mentioning that the hybrid algorithms proposed in $[12,18,22]$ are computationally complex. Therefore, it is natural to have improved and computationally simpler counterparts.

Quite recently, Zuo et al. [16] proposed a hybrid algorithm for total quasi- $\varphi$-asymptotically nonexpansive mappings and established strong convergence results in a Banach space. Moreover, they characterized such strong convergence results by using the notion of Mosco convergence; see also [23]. Inspired and motivated by the work of Takahashi and Zembayashi [21], Chang et al. [12] and Zuo et al. [16], we aim to introduce and analyze a general algorithm based on the shrinking projection method for finding a common element of the set of common solutions of a finite family of generalized equilibrium problems and the set of common fixed points of two countable families of total quasi- $\varphi$ asymptotically nonexpansive mappings. We also characterize the set of common solutions for families of total quasi- $\varphi$-asymptotically nonexpansive mappings and equilibrium problems in terms of Mosco convergence.

We now introduce the notion of Mosco convergence.

Let $\left\{C_{n}\right\}$ be a sequence of nonempty closed convex subsets of a reflexive Banach space $E$. We denote the set of all strong limit points of $\left\{C_{n}\right\}$ by $s-L i_{n} C_{n}$, that is, $x \in s-L i_{n} C_{n}$ if and only if there exists $\left\{x_{n}\right\} \subset E$ such that $\left\{x_{n}\right\}$ converges strongly to $x$ and that $x_{n} \in C_{n}$ for all $n$. Similarly, we define the set of all weak subsequential limit points by $w-L s_{n} C_{n}$; $y \in w-L s_{n} C_{n}$ if and only if there exist a subsequence $\left\{C_{n_{i}}\right\}$ of $\left\{C_{n}\right\}$ and a sequence $\left\{y_{i}\right\} \subset E$ such that $\left\{y_{i}\right\}$ converges weakly to $y$ and $y_{i} \in C_{n_{i}}$ for all $i$. If $C_{0}$ satisfies $C_{0}=s-L i_{n} C_{n}=$ $w-L s_{n} C_{n}$, then we say that $\left\{C_{n}\right\}$ converges to $C_{0}$ in the sense of Mosco and we write $C_{0}=M-\lim _{n} C_{n}$. By definition, it always holds that $s-L i_{n} C_{n} \subset w-L s_{n} C_{n}$. Therefore, to prove $C_{0}=M-\lim _{n} C_{n}$, it suffices to show that $w-L s_{n} C_{n} \subset C_{0} \subset s-L i_{n} C_{n}$. One of the simplest examples of Mosco convergence is a decreasing sequence $\left\{C_{n}\right\}$ with respect to inclusion. The Mosco limit of such a sequence is $\bigcap_{n=1}^{\infty} C_{n}$. For more details, we refer to $[23,24]$.

For a relation between a sequence of closed convex sets and the corresponding generalized projections, we state the following lemma which plays a key role in our main result. 
Lemma 1.1 ([23], Theorem 2.2) Let E be a smooth, reflexive and strictly convex Banach space having the Kadec-Klee property. Let $\left\{C_{n}\right\}$ be a sequence of nonempty closed subset of $E$. If $C_{0}=M-\lim _{n} C_{n}$ exists and is nonempty, then $\left\{\Pi_{C_{n}} x\right\}$ converges strongly to $\Pi_{C_{0}} x$ for each $x \in C$.

The following two results can be found as Remark 7.3 in [5].

Lemma 1.2 Let $C$ be a nonempty closed convex subset of a smooth, strictly convex and reflexive Banach space $E$, let $x_{0} \in E$ and let $x \in C$. Then $\Pi_{C} x_{0}=x$ if and only if

$$
\left\langle x-y, J x_{0}-J x\right\rangle \geq 0 \text { for all } y \in C .
$$

Lemma 1.3 Let $E$ be a reflexive, strictly convex and smooth Banach space, let $C$ be a nonempty closed convex subset of $E$ and let $x \in E$. Then

$$
\varphi\left(y, \Pi_{C} x\right)+\varphi\left(\Pi_{C} x, x\right) \leq \varphi(y, x) \quad \text { for all } y \in C .
$$

The following well-known results are also needed in the sequel for the development of our main result.

Lemma 1.4 ([25], Proposition 2) Let E be a uniformly convex and smooth Banach space and let $\left\{x_{n}\right\},\left\{y_{n}\right\}$ be two sequences of $E$. If $\varphi\left(x_{n}, y_{n}\right) \rightarrow 0$ and either $\left\{x_{n}\right\}$ or $\left\{y_{n}\right\}$ is bounded, then $x_{n}-y_{n} \rightarrow 0$.

Lemma 1.5 ([6], Lemma 1.4) Let $E$ be a uniformly convex Banach space and let $B_{r}[0]$ be a closed ball in $E$. Then there exists a continuous strictly increasing convex function $g:[0, \infty) \rightarrow[0, \infty)$ with $g(0)=0$ such that

$$
\|\alpha x+\beta y+\gamma z\|^{2} \leq \alpha\|x\|^{2}+\beta\|y\|^{2}+\gamma\|z\|^{2}-\alpha \beta g(\|x-y\|)
$$

for all $x, y \in B_{r}[0]$ and $\alpha, \beta, \gamma \in[0,1]$ with $\alpha+\beta+\gamma=1$.

For solving the equilibrium problem, let us assume that the bifunction $f$ satisfies the following conditions $(c f .[2,26])$ :

(A1) $f(x, x)=0$ for all $x \in C$;

(A2) $f$ is monotone, i.e., $f(x, y)+f(y, x) \leq 0$ for all $x, y \in C$;

(A3) $\lim \sup _{t \downarrow} f(t z+(1-t) x, y) \leq f(x, y)$ for all $x, y, z \in C$;

(A4) $f(x, \cdot)$ is convex and lower semicontinuous for all $x \in C$.

The following result is stated as Lemma 2.7 in [21] (see also [2]).

Lemma 1.6 Let $C$ be a closed convex subset of a smooth, strictly convex and reflexive Banach space $E$, let $f: C \times C \rightarrow \mathbb{R}$ be a bifunction satisfying (A1)-(A4), let $r>0$ and $x \in E$. Then there exists $z \in C$ such that

$$
f(z, y)+\frac{1}{r}\langle y-z, J z-J x\rangle \geq 0 \quad \text { for all } y \in C .
$$


Lemma 1.7 ([21], Lemma 2.8) Let $C$ be a closed convex subset of a uniformly smooth, strictly convex and reflexive Banach space E. Let $f: C \times C \rightarrow \mathbb{R}$ be a bifunction satisfying (A1)-(A4). For $r>0$ and $x \in E$, define a mapping $V_{r}: E \rightarrow C$ by

$$
V_{r}(x)=\left\{z \in C: f(z, y)+\frac{1}{r}\langle y-z, J z-J x\rangle \geq 0 \text { for all } y \in C\right\}
$$

for all $x \in C$. Then the following hold:

(1) $E P(f)$ is closed and convex;

(2) $V_{r}$ is single-valued;

(3) $V_{r}$ is firmly nonexpansive-type mapping, i.e.,

$$
\left\langle V_{r} x-V_{r} y, J V_{r} x-J V_{r} y\right\rangle \leq\left\langle V_{r} x-V_{r} y, J x-J y\right\rangle \quad \text { for all } x, y \in E,
$$

(4) $F\left(V_{r}\right)=E P(f)$.

\section{Main results}

Let $C$ be a nonempty closed convex subset of a strictly convex, reflexive and smooth Banach space $E$ having the Kadec-Klee property. Let $T_{i}, S_{i}: C \rightarrow C$ be two countable families of uniformly $L$-Lipschitzian and uniformly total quasi- $\varphi$-asymptotically nonexpansive mappings with sequences $\left\{k_{n}^{T}\right\},\left\{\eta_{n}^{T}\right\}$ and $\left\{k_{n}^{S}\right\},\left\{\eta_{n}^{S}\right\}$, respectively. Let $f_{n}: C \times C \rightarrow \mathbb{R}$, $n=1,2,3, \ldots, N$, be a finite family of bifunctions such that $f_{n} \equiv f_{n}(\bmod N)$ (here the $\bmod N$ function takes values in $\{1,2,3, \ldots, N\})$.

\section{Algorithm}

$$
\left\{\begin{array}{l}
x_{0} \in C_{0}=C, \\
y_{n, i}=J^{-1}\left(\alpha_{n, i} J x_{n}+\beta_{n, j} J T_{i}^{n} x_{n}+\gamma_{n, i} J S_{i}^{n} x_{n}\right), \\
f_{n}\left(u_{n, i}, y\right)+\left\langle A_{n} u_{n, i}, y-u_{n, i}\right\rangle+\frac{1}{r_{n}}\left\langle y-u_{n, i} J u_{n, i}-J y_{n, i}\right\rangle \geq 0 \quad \text { for all } y \in C, \\
C_{n+1}=\left\{z \in C_{n}: \sup _{i \geq 1} \varphi\left(z, u_{n, i}\right) \leq \varphi\left(z, x_{n}\right)+\Theta_{n}\right\}, \\
x_{n+1}=\Pi_{C_{n+1}} x_{0}, \quad n \geq 0,
\end{array}\right.
$$

where $\alpha_{n, i}+\beta_{n, i}+\gamma_{n, i}=1$ and $\Theta_{n}:=\left(1-\alpha_{n, i}\right)\left\{k_{n} \sup _{u \in F} \xi\left(\varphi\left(u, x_{n}\right)\right)+\eta_{n}\right\}$.

Theorem 2.1 Let $C$ be a nonempty closed convex subset of a strictly convex, reflexive and smooth Banach space $E$ having the Kadec-Klee property and let $T_{i}, S_{i}: C \rightarrow C$ be two countable families of uniformly L-Lipschitzian and uniformly total quasi- $\varphi$-asymptotically nonexpansive mappings with sequences $\left\{k_{n}\right\}$ and $\left\{\eta_{n}\right\}$, where $0<L:=\max \left\{L_{i}^{T}, L_{i}^{S}\right\}$ for each $i \geq 1,\left\{k_{n}\right\}:=\max _{n \geq 1}\left\{\left\{k_{n}^{T}\right\},\left\{k_{n}^{S}\right\}\right\}$ and $\left\{\eta_{n}\right\}:=\max _{n \geq 1}\left\{\left\{\eta_{n}^{T}\right\},\left\{\eta_{n}^{S}\right\}\right\}$. Let $f_{n}: C \times C \rightarrow \mathbb{R}, n=$ $1,2,3, \ldots, N$, be a finite family of bifunctions satisfying (A1)-(A4) such that $f_{n}=f_{n}(\bmod N)$. Let $a \leq \alpha_{n, i}, \beta_{n, i}, \gamma_{n, i} \leq b$ for some $a, b \in(0,1)$ and $\left\{r_{n}\right\} \subset(0, \infty)$ satisfying:

(C1) $\liminf _{n \rightarrow \infty} r_{n}>0$.

Assume that $F:=\left[\bigcap_{i=1}^{\infty} F\left(T_{i}\right)\right] \cap\left[\bigcap_{i=1}^{\infty} F\left(S_{i}\right)\right] \cap\left[\bigcap_{n=1}^{N} G E P\left(f_{n}\right)\right] \neq \emptyset$. Then the sequence $\left\{x_{n}\right\}$ generated by (2.1) converges strongly to $x=\Pi_{F} x_{0}$, where $\Pi_{F}$ is the generalized projection of E onto F.

Proof For each $n=1,2,3, \ldots, N$, define $G_{n}: C \times C \rightarrow \mathbb{R}$ by $G_{n}(x, y)=f_{n}(x, y)+\left\langle A_{n} x, y-x\right\rangle$. Then $G_{n}$ coincides with the classical equilibrium problem and satisfies (A1)-(A4). Now, 
we show that algorithm (2.1) is well defined. By Lemma 1.7(1) we have that $F$ is closed and convex. Next we show that $C_{n+1}$ is closed and convex. Clearly, $C_{0}=C$ is closed and convex. Suppose that $C_{k}$ is closed and convex for $k \in \mathbb{N}$ (set of naturals). For each $z \in C_{k}$, we observe that

$$
\begin{aligned}
C_{k+1} & =\left\{z \in C_{k}: \sup _{i \geq 1} \varphi\left(z, u_{k, i}\right) \leq \varphi\left(z, x_{k}\right)+\Theta_{k}\right\} \\
& =\bigcap_{i \geq 1}\left\{z \in C_{k}: \varphi\left(z, u_{k, i}\right) \leq \varphi\left(z, x_{k}\right)+\Theta_{k}\right\} \\
& =\bigcap_{i \geq 1}\left\{z \in C_{k}: 2\left\langle z, J x_{k}-J u_{k, i}\right\rangle+\left\|u_{k, i}\right\|^{2}-\left\|x_{k}\right\|^{2}-\Theta_{k} \leq 0\right\} \cap C_{k} .
\end{aligned}
$$

This implies that $C_{k+1}$ is closed and convex. This implies, inductively, that $C_{n+1}$ is closed and convex for all $n \geq 0$. Now we prove that $F \subset C_{n+1}$ for each $n \geq 0$. Obviously, $F \subset C_{0}=$ $C$. Suppose that $F \subset C_{k}$ for some $k \in \mathbb{N}$. It follows from Lemma 1.6 that $u_{k, i}=V_{r_{k}} y_{k, i}$ and $V_{r_{k}}$ is relatively nonexpansive. Hence, for any $p \in F$, we have

$$
\begin{aligned}
\varphi\left(p, u_{k, i}\right)= & \varphi\left(p, V_{r_{k}} y_{k, i}\right) \leq \varphi\left(p, y_{k, i}\right) \\
= & \varphi\left(p, J^{-1}\left(\alpha_{k, i} J x_{k}+\beta_{k, i} J T_{i}^{n} x_{k}+\gamma_{k, i} J S_{i}^{n} x_{k}\right)\right) \\
= & \|p\|^{2}-2\left\langle p, \alpha_{k, i} J x_{k}+\beta_{k, i} J T_{i}^{n} x_{k}+\gamma_{k, i} J S_{i}^{n} x_{k}\right\rangle \\
& +\left\|\alpha_{k, i} J x_{k}+\beta_{k, i} J T_{i}^{n} x_{k}+\gamma_{k, i} J S_{i}^{n} x_{k}\right\|^{2} \\
\leq & \|p\|^{2}-2 \alpha_{k, i}\left\langle p, J x_{k}\right\rangle-2 \beta_{k, i}\left\langle p, J T_{i}^{n} x_{k}\right\rangle-2 \gamma_{k, i}\left\langle p, J S_{i}^{n} x_{k}\right\rangle \\
& +\alpha_{k, i}\left\|x_{k}\right\|^{2}+\beta_{k, i}\left\|T_{i}^{n} x_{k}\right\|^{2}+\gamma_{k, i}\left\|S_{i}^{n} x_{k}\right\|^{2} \\
= & \alpha_{k, i}\left(\|p\|^{2}-2\left\langle p, J x_{k}\right\rangle+\left\|x_{k}\right\|^{2}\right) \\
& +\beta_{k, i}\left(\|p\|^{2}-2\left\langle p, J T_{i}^{n} x_{k}\right\rangle+\left\|T_{i}^{n} x_{k}\right\|^{2}\right) \\
& +\gamma_{k, i}\left(\|p\|^{2}-2\left\langle p, J S_{i}^{n} x_{k}\right\rangle+\left\|S_{i}^{n} x_{k}\right\|^{2}\right) \\
= & \alpha_{k, i} \varphi\left(p, x_{k}\right)+\beta_{k, i} \varphi\left(p, T_{i}^{n} x_{k}\right)+\gamma_{k, i} \varphi\left(p, S_{i}^{n} x_{k}\right) \\
\leq & \alpha_{k, i} \varphi\left(p, x_{k}\right)+\beta_{k, i}\left\{\varphi\left(p, x_{k}\right)+k_{k} \xi\left(\varphi\left(p, x_{k}\right)\right)+\eta_{k}\right\} \\
& +\gamma_{k, i}\left\{\varphi\left(p, x_{k}\right)+k_{k} \xi\left(\varphi\left(p, x_{k}\right)\right)+\eta_{k}\right\} .
\end{aligned}
$$

That is,

$$
\varphi\left(p, u_{k, i}\right) \leq \varphi\left(p, x_{k}\right)+\left(1-\alpha_{k, i}\right)\left\{k_{k} \xi\left(\varphi\left(p, x_{k}\right)\right)+\eta_{k}\right\}=\varphi\left(p, x_{k}\right)+\Theta_{k},
$$

where $\Theta_{k}:=\left(1-\alpha_{k, i}\right)\left\{k_{k} \sup _{u \in F} \xi\left(\varphi\left(u, x_{k}\right)\right)+\eta_{k}\right\}$. This shows that $p \in C_{k+1}$; consequently, $F \subset C_{k+1}$. By induction, we also get that $F \subset C_{n+1}$ for all $n \geq 0$ with $\Theta_{n}:=\left(1-\alpha_{n, i}\right) \times$ $\left\{k_{n} \sup _{u \in F} \xi\left(\varphi\left(u, x_{n}\right)\right)+\eta_{n}\right\}$. Since $F \neq \emptyset$ and $C_{n+1}$ is a nonempty closed convex subset of $E$, hence both $\Pi_{C_{n+1}} x$ and $\left\{x_{n}\right\}$ are well defined.

Note that $\varphi\left(x_{n}, x^{*}\right)$ is nondecreasing. In fact, from the definition of $C_{n+1}$, we conclude that $x_{n}=\Pi_{C_{n}} x_{0}$ and $x_{n+1}=\Pi_{C_{n+1}} x_{0} \in C_{n+1} \subset C_{n}$; hence

$$
\varphi\left(x_{n}, x^{*}\right) \leq \varphi\left(x_{n+1}, x^{*}\right) .
$$


Moreover, from Lemma 1.3 we get that

$$
\varphi\left(x_{n}, x^{*}\right)=\varphi\left(\Pi_{C_{n}} x_{0}, x^{*}\right) \leq \varphi\left(p, x^{*}\right)-\varphi\left(p, \Pi_{C_{n}} x_{0}\right) \leq \varphi\left(p, x^{*}\right)
$$

for each $p \in F$.

So $\varphi\left(x_{n}, x^{*}\right)$ is nondecreasing and bounded. This implies that $\lim _{n \rightarrow \infty} \varphi\left(x_{n}, x^{*}\right)$ exists.

Let $m \in \mathbb{Z}^{+}$(a set of positive integers). Then Lemma 1.3 implies

$$
\begin{aligned}
\varphi\left(x_{n+m}, x_{n}\right) & =\varphi\left(x_{n+m}, \Pi_{C_{n}} x_{0}\right) \leq \varphi\left(x_{n+m}, x^{*}\right)-\varphi\left(\Pi_{C_{n}} x_{0}, x^{*}\right) \\
& =\varphi\left(x_{n+m}, x^{*}\right)-\varphi\left(x_{n}, x^{*}\right) .
\end{aligned}
$$

Letting $n \rightarrow \infty$, we have $\varphi\left(x_{n+m}, x_{n}\right) \rightarrow 0$. By Lemma 1.4, we have

$$
\left\|x_{n+m}-x_{n}\right\| \stackrel{n \rightarrow \infty}{\rightarrow} 0
$$

Hence $\left\{x_{n}\right\}$ is Cauchy. Since $C$ is a closed subset of the Banach space $E$, we can assume that there exists a point $x \in C$ such that

$$
x_{n} \rightarrow x \quad \text { as } n \rightarrow \infty .
$$

Note that $\left\{C_{n}\right\}$ is a decreasing sequence of closed convex subsets of $E$ with $C_{0}=\bigcap_{n=1}^{\infty} C_{n}$ is nonempty. That is,

$$
M-\lim _{n} C_{n}=C_{0}=\bigcap_{n=1}^{\infty} C_{n} \neq \emptyset .
$$

Hence Lemma 1.1 asserts that $\left\{\Pi_{C_{n}} x_{0}\right\}$ converges to $\Pi_{C_{0}} x_{0}$.

In what follows, we show that:

Step 1. $x \in\left[\bigcap_{i=1}^{\infty} F\left(T_{i}\right)\right] \cap\left[\bigcap_{i=1}^{\infty} F\left(S_{i}\right)\right]$

Step 2. $x \in\left[\bigcap_{n=1}^{N} E P\left(G_{n}\right)\right]$;

Step 3. $x=\Pi_{F} x_{0}$.

Proof of Step 1. As $x_{n+1} \in C_{n+1}$, so $\sup _{i \geq 1} \varphi\left(x_{n+1}, u_{n, i}\right) \leq \varphi\left(x_{n+1}, x_{n}\right)+\Theta_{n}$. It follows from (2.3) and the fact that $\Theta_{n} \stackrel{n \rightarrow \infty}{\rightarrow} 0$, we get $\lim _{n \rightarrow \infty} \varphi\left(x_{n+1}, u_{n, i}\right)=0$ for all $i \geq 1$. Again by Lemma 1.4, we have

$$
\lim _{n \rightarrow \infty}\left\|x_{n+1}-u_{n, i}\right\|=0, \quad i \geq 1 .
$$

Observe that the following implication of the triangle inequality

$$
\left\|x_{n}-u_{n, i}\right\| \leq\left\|x_{n}-x_{n+1}\right\|+\left\|x_{n+1}-u_{n, i}\right\|
$$

yields that

$$
\lim _{n \rightarrow \infty}\left\|x_{n}-u_{n, i}\right\|=0
$$


As $J$ is uniformly norm-to-norm continuous on bounded sets, so we have

$$
\lim _{n \rightarrow \infty}\left\|J x_{n}-J u_{n, i}\right\|=0
$$

Moreover, it follows from (2.4) and (2.6) that

$$
\lim _{n \rightarrow \infty}\left\|u_{n, i}-x\right\|=0 \quad \text { for all } i \geq 1 .
$$

From (2.2), we know that $\varphi\left(p, y_{n, i}\right) \leq \varphi\left(p, x_{n}\right)+\Theta_{n}$ for all $i \geq 1$ and for all $p \in F$. So, by Lemma 1.3, we have

$$
\begin{aligned}
\varphi\left(u_{n, i}, y_{n, i}\right) & =\varphi\left(V_{r_{n}} y_{n, i}, y_{n, i}\right) \leq \varphi\left(p, y_{n, i}\right)-\varphi\left(p, V_{r_{n}} y_{n, i}\right) \\
& \leq \varphi\left(p, x_{n}\right)+\Theta_{n}-\varphi\left(p, V_{r_{n}} y_{n, i}\right) \\
& =\varphi\left(p, x_{n}\right)-\varphi\left(p, u_{n, i}\right)+\Theta_{n} \\
& =\left\|x_{n}\right\|^{2}-\left\|u_{n, i}\right\|^{2}-2\left\langle p, J x_{n}-J u_{n, i}\right\rangle+\Theta_{n} \\
& \leq\left\|x_{n}-u_{n, i}\right\|\left(\left\|x_{n}\right\|+\left\|u_{n, i}\right\|\right)-2\left\langle p, J x_{n}-J u_{n, i}\right\rangle+\Theta_{n} .
\end{aligned}
$$

Letting $n \rightarrow \infty$ in the above estimate and using (2.7), we have $\lim _{n \rightarrow \infty} \varphi\left(u_{n, i}, y_{n, i}\right)=0$ for all $i \geq 1$; consequently, Lemma 1.4 asserts that

$$
\lim _{n \rightarrow \infty}\left\|u_{n, i}-y_{n, i}\right\|=0, \quad i \geq 1 .
$$

From (2.6) and (2.9), we have

$$
\lim _{n \rightarrow \infty}\left\|x_{n}-y_{n, i}\right\|=0, \quad i \geq 1 .
$$

Hence, we conclude that

$$
y_{n, i} \stackrel{n \rightarrow \infty}{\rightarrow} x \quad \text { for all } i \geq 1
$$

On the other hand, from Lemma 1.5, we have

$$
\begin{aligned}
\varphi\left(p, u_{n, i}\right)= & \varphi\left(p, V_{r_{n}} y_{n, i}\right) \leq \varphi\left(p, y_{n, i}\right) \\
= & \varphi\left(p, J^{-1}\left(\alpha_{n, i} J x_{n}+\beta_{n, i} J T_{i}^{n} x_{n}+\gamma_{n, i} J S_{i}^{n} x_{n}\right)\right) \\
= & \|p\|^{2}-2\left\langle p, \alpha_{n, i} J x_{n}+\beta_{n, i} J T_{i}^{n} x_{n}+\gamma_{n, i} J S_{i}^{n} x_{n}\right\rangle \\
& +\left\|\alpha_{n, i} J x_{n}+\beta_{n, i} J T_{i}^{n} x_{n}+\gamma_{n, i} J S_{i}^{n} x_{n}\right\|^{2} \\
\leq & \|p\|^{2}-2 \alpha_{n, i}\left\langle p, J x_{n}\right\rangle-2 \beta_{n, i}\left\langle p, J T_{i}^{n} x_{n}\right\rangle-2 \gamma_{n, i}\left(p, J S_{i}^{n} x_{n}\right\rangle \\
& +\alpha_{n, i}\left\|x_{n}\right\|^{2}+\beta_{n, i}\left\|T_{i}^{n} x_{n}\right\|^{2}+\gamma_{n, i}\left\|S_{i}^{n} x_{n}\right\|^{2}-\alpha_{n, i} \beta_{n, i} g\left(\left\|J x_{n}-J T_{i}^{n} x_{n}\right\|\right) \\
= & \alpha_{n, i} \varphi\left(p, x_{n}\right)+\beta_{n, i} \varphi\left(p, T_{i}^{n} x_{n}\right)+\gamma_{n, i} \varphi\left(p, S_{i}^{n} x_{n}\right) \\
& -\alpha_{n, i} \beta_{n, i} g\left(\left\|J x_{n}-J T_{i}^{n} x_{n}\right\|\right) \\
\leq & \alpha_{n, i} \varphi\left(p, x_{n}\right)+\left(1-\alpha_{n, i}\right) \varphi\left(p, x_{n}\right)+\left(1-\alpha_{n, i}\right) \Theta_{n}-\alpha_{n, i} \beta_{n, i} g\left(\left\|J x_{n}-J T_{i}^{n} x_{n}\right\|\right) .
\end{aligned}
$$


Since $a \leq \alpha_{n, i}, \beta_{n, i}$, re-arranging the terms of the above estimate and simplifying, we get

$$
a^{2} g\left(\left\|J x_{n}-J T_{i}^{n} x_{n}\right\|\right) \leq \varphi\left(p, x_{n}\right)-\varphi\left(p, u_{n, i}\right)+\left(1-\alpha_{n, i}\right) \Theta_{n} .
$$

Reasoning as above and then utilizing (2.6), we get that

$$
\lim _{n \rightarrow \infty} g\left(\left\|J x_{n}-J T_{i}^{n} x_{n}\right\|\right)=0, \quad i \geq 1 .
$$

Note that $g$ is a continuous function and $g(0)=0$, so we have

$$
\lim _{n \rightarrow \infty}\left\|J x_{n}-J T_{i}^{n} x_{n}\right\|=0, \quad i \geq 1
$$

In view of estimate (2.4), we conclude that $J x_{n} \stackrel{n \rightarrow \infty}{\rightarrow} J x$. Hence, from (2.11) we obtain

$$
J T_{i}^{n} x_{n} \stackrel{n \rightarrow \infty}{\rightarrow} J x, \quad i \geq 1
$$

Since $J^{-1}$ is uniformly norm-to-norm continuous, (2.11) implies that

$$
\lim _{n \rightarrow \infty}\left\|x_{n}-T_{i}^{n} x_{n}\right\|=0, \quad i \geq 1 .
$$

Observe that

$$
\left\|T_{i}^{n} x_{n}-x\right\| \leq\left\|T_{i}^{n} x_{n}-x_{n}\right\|+\left\|x_{n}-x\right\| .
$$

So, we conclude from (2.4) and (2.13) that

$$
\lim _{n \rightarrow \infty}\left\|T_{i}^{n} x_{n}-x\right\|=0, \quad i \geq 1 .
$$

Since each $T_{i}$ is uniformly $L$-Lipschitzian, we have

$$
\begin{aligned}
\left\|T_{i}^{n+1} x_{n}-T_{i}^{n} x_{n}\right\| \leq & \left\|T_{i}^{n+1} x_{n}-T_{i}^{n+1} x_{n+1}\right\|+\left\|T_{i}^{n+1} x_{n+1}-x_{n+1}\right\| \\
& +\left\|x_{n+1}-x_{n}\right\|+\left\|x_{n}-T_{i}^{n} x_{n}\right\| \\
\leq & (L+1)\left\|x_{n+1}-x_{n}\right\|+\left\|T_{i}^{n+1} x_{n+1}-x_{n+1}\right\|+\left\|x_{n}-T_{i}^{n} x_{n}\right\| .
\end{aligned}
$$

Hence, from (2.3) and (2.13), we obtain that

$$
\lim _{n \rightarrow \infty}\left\|T_{i}^{n+1} x_{n}-T_{i}^{n} x_{n}\right\|=0, \quad i \geq 1 .
$$

Moreover, it yields that $\lim _{n \rightarrow \infty}\left\|T_{i}^{n+1} x_{n}-x\right\|=0, i \geq 1$; consequently, we have $T_{i}\left(T_{i}^{n} x_{n}\right) \stackrel{n \rightarrow \infty}{\rightarrow} x, i \geq 1$. So we infer that $x \in \bigcap_{i=1}^{\infty} F\left(T_{i}\right)$.

Reasoning as above, one can also show that $x \in \bigcap_{i=1}^{\infty} F\left(S_{i}\right)$.

Proof of Step 2. We first show that $x \in E P\left(G_{1}\right)$, where $G_{1}=G_{n_{j}}$ for some $j \geq 1$. In view of estimate (2.9) and (C1), i.e., $\liminf _{n \rightarrow \infty} r_{n}>0$, we observe that

$$
\lim _{j \rightarrow \infty} \frac{\left\|J u_{n_{j}, i}-J y_{n_{j}, i}\right\|}{r_{n_{j}}}=0, \quad i \geq 1 .
$$


From $u_{n_{j}, i}=V_{r_{n_{j}}} y_{n_{j}, i}$, for all $n \geq 0$ and $i \geq 1$, we have

$$
G_{1}\left(u_{n_{j}, i}, y\right)+\frac{1}{r_{n_{j}}}\left\langle y-u_{n_{j}, i}, J u_{n_{j}, i}-J y_{n_{j}, i}\right\rangle \geq 0 \quad \text { for all } y \in C \text {. }
$$

Using (A2), the above estimate yields that

$$
\frac{1}{r_{n_{j}}}\left\langle y-u_{n_{j}, i}, J u_{n_{j}, i}-J y_{n, i}\right\rangle \geq-G_{1}\left(u_{n_{j}, i}, y\right) \geq G_{1}\left(y, u_{n_{j}, i}\right) \quad \text { for all } y \in C \text {. }
$$

From $u_{n_{j}, i} \rightarrow x$ and (A4), we obtain $G_{1}(y, x) \leq 0$ for all $y \in C$. Let $y_{t}=t y+(1-t) x_{0}$ for $0<t<1$ and $y \in C$. Then $y_{t} \in C$ and hence $G_{1}\left(y_{t}, x\right) \leq 0$. From (A1) and (A4), we have $0=G_{1}\left(y_{t}, y_{t}\right) \leq t G_{1}\left(y_{t}, y\right)+(1-t) G_{1}\left(y_{t}, x\right) \leq t G_{1}\left(y_{t}, y\right)$. Thus, $G_{1}\left(y_{t}, y\right) \geq 0$. From (A3), we have $G_{1}(x, y) \geq 0$ for all $y \in C$. Hence $x \in E P\left(G_{1}\right)$. In a similar fashion, we have some $k \geq 1$ such that $G_{2}=G_{n_{k}}$ and $x \in E P\left(G_{2}\right)$. Therefore, $x \in \bigcap_{n=1}^{N} E P\left(G_{n}\right)$ and hence $x \in F$.

Proof of Step 3. Lemma 1.1 asserts that the sequence $\left\{x_{n}\right\}=\left\{\Pi_{C_{n}} x_{0}\right\}$ converges to $\Pi_{C_{0}} x_{0}$. Let $p=\Pi_{C_{0}} x_{0} \in F$ and $F$ is a nonempty closed convex subset of $C_{0}=\bigcap_{n=1}^{\infty} C_{n}$. Therefore $p=\Pi_{F} x_{0}$. It suffices to show that $x=p$. For this, we reason as follows:

Since $x_{n}=\Pi_{C_{n}} x_{0}$ and $p \in F \subset C_{n}$, we have

$$
\varphi\left(x_{n}, x_{0}\right) \leq \varphi\left(p, x_{0}\right)
$$

Since the norm is weakly lower semicontinuous, we have

$$
\begin{aligned}
\varphi\left(x, x_{0}\right) & =\|x\|^{2}-2\left\langle x, J x_{0}\right\rangle+\left\|x_{0}\right\|^{2} \\
& \leq \liminf _{k \rightarrow \infty}\left(\left\|x_{n_{k}}\right\|^{2}-2\left\langle x_{n_{k}}, J x_{0}\right\rangle+\left\|x_{0}\right\|^{2}\right) \\
& \leq \liminf _{k \rightarrow \infty} \varphi\left(x_{n_{k}}, x_{0}\right) \\
& \leq \limsup _{k \rightarrow \infty} \varphi\left(x_{n_{k}}, x_{0}\right) \leq \varphi\left(p, x_{0}\right) .
\end{aligned}
$$

From the definition of $\Pi_{F}$, we have $x=p$. Hence $\lim _{k \rightarrow \infty} \varphi\left(x_{n_{k}}, x_{0}\right)=\varphi\left(p, x_{0}\right)$. Therefore, we have

$$
\begin{aligned}
0 & =\lim _{k \rightarrow \infty}\left(\varphi\left(x_{n_{k}}, x_{0}\right)-\varphi\left(x, x_{0}\right)\right) \\
& =\lim _{k \rightarrow \infty}\left(\left\|x_{n_{k}}\right\|^{2}-\|x\|^{2}+2\left\langle x_{n_{k}}-x, J x_{0}\right\rangle\right) \\
& =\lim _{k \rightarrow \infty}\left(\left\|x_{n_{k}}\right\|^{2}-\|x\|^{2}\right) .
\end{aligned}
$$

Since $E$ has the Kadec-Klee property, we have that $\lim _{k \rightarrow \infty} x_{n_{k}}=x=\Pi_{F} x_{0}$.

The arbitrariness of $\left\{x_{n_{k}}\right\}$ implies that $\left\{x_{n}\right\}$ converges strongly to $x=\Pi_{F} x_{0}$.

Remark 2.2 If $\left\{T_{i}\right\}_{i=1}^{N}$ and $\left\{S_{i}\right\}_{i=1}^{N}$ are finite families in Theorem 2.1, then its conclusion can be strengthened as follows:

The sequence $\left\{x_{n}\right\}$ generated by (2.1) converges strongly to some $x \in F$ if and only if $\lim _{n \rightarrow \infty} d\left(x_{n}, F\right)=0$, where $x=\Pi_{F} x_{0}$ and $d(q, F)=\inf \{d(q, y): y \in F\}$. 
Proof The necessity is obvious. Conversely, suppose that $\lim _{n \rightarrow \infty} d\left(x_{n}, F\right)=0$. Since $\varphi\left(x_{n}, x^{*}\right)$ is bounded and $\lim _{n \rightarrow \infty} \varphi\left(x_{n}, x^{*}\right)$ exists (established above). Moreover, Lemma 1.3 and the following estimate

$$
\begin{aligned}
\varphi\left(x_{n+m}, x_{n}\right) & =\varphi\left(x_{n+m}, \Pi_{C_{n}} x\right) \leq \varphi\left(x_{m}, x^{*}\right)-\varphi\left(\Pi_{C_{n}} x, x^{*}\right) \\
& =\varphi\left(x_{n+m}, x^{*}\right)-\varphi\left(x_{n}, x^{*}\right)
\end{aligned}
$$

imply $\varphi\left(x_{n+m}, x_{n}\right) \rightarrow 0$ when $m, n \rightarrow \infty$. Hence, Lemma 1.4 asserts that $\left\|x_{n+m}-x_{n}\right\| \rightarrow 0$. Thus, $\left\{x_{n}\right\}$ is a Cauchy sequence. Therefore, there exists a point $x \in C$ such that $x_{n} \rightarrow x$ as $n \rightarrow \infty$. Thus,

$$
d(x, F)=\lim _{n \rightarrow \infty} d\left(x_{n}, F\right)=0 .
$$

Since $F$ is closed, this implies $x \in F$. Now, as in the proof of Step 3 in Theorem 2.1, we have $x=\Pi_{F} x_{0}$.

To proceed further, we need the following concept.

Let $f$ be a nondecreasing self-mapping on $[0, \infty)$ with $f(0)=0$ and $f(t)>0$ for all $t \in$ $(0, \infty)$. Let $\left\{T_{i}: i \in I\right\}$ and $\left\{S_{i}: i \in I\right\}$ be two finite families of total quasi- $\varphi$-asymptotically nonexpansive mappings on $C$ with $F \neq \emptyset$. Then the two families are said to satisfy condition (I) on $C$ if

$$
\|x-T x\| \geq f(d(x, F)) \quad \text { or } \quad\|x-S x\| \geq f(d(x, F)) \quad \text { for all } x \in C .
$$

As an application of Theorem 2.1 to the case of finite families of mappings $\left\{T_{i}\right\}_{i=1}^{N}$ and $\left\{S_{i}\right\}_{i=1}^{N}$, we obtain the following new strong convergence result.

Theorem 2.3 Let $C$ be a nonempty closed convex subset of a strictly convex, reflexive and smooth Banach space E having the Kadec-Klee property and let $T_{i}, S_{i}: C \rightarrow C$ be two finite families of uniformly L-Lipschitzian and uniformly total quasi- $\varphi$-asymptotically nonexpansive mappings with sequences $\left\{k_{n}\right\}$ and $\left\{\eta_{n}\right\}$, where $0<L:=\max \left\{L_{i}^{T}, L_{i}^{S}\right\}$ for each $i \geq 1,\left\{k_{n}\right\}:=\max _{n \geq 1}\left\{\left\{k_{n}^{T}\right\},\left\{k_{n}^{S}\right\}\right\}$ and $\left\{\eta_{n}\right\}:=\max _{n \geq 1}\left\{\left\{\eta_{n}^{T}\right\},\left\{\eta_{n}^{S}\right\}\right\}$. Let $f_{n}: C \times C \rightarrow \mathbb{R}, n=$ $1,2,3, \ldots, N$, be a finite family of bifunctions satisfying (A1)-(A4) such that $f_{n}=f_{n}(\bmod N)$. Let $a \leq \alpha_{n, i}, \beta_{n, i}, \gamma_{n, i} \leq$ for some $a, b \in(0,1)$ and $\left\{r_{n}\right\} \subset(0, \infty)$ satisfying

(C1) $\liminf _{n \rightarrow \infty} r_{n}>0$.

Assume that $T_{i}$ and $S_{i}$ satisfy condition (I) and $F \neq \emptyset$. Then the sequence $\left\{x_{n}\right\}$ generated by (2.1) converges strongly to some $x \in F$, where $x=\Pi_{F} x_{0}$.

Proof It follows from Theorem 2.1 that

$$
\lim _{n \rightarrow \infty}\left\|x_{n}-T_{i}^{n} x_{n}\right\|=0=\lim _{n \rightarrow \infty}\left\|x_{n}-S_{i}^{n} x_{n}\right\|
$$

Since $\left\{T_{i}^{n}: i \in I\right\}$ and $\left\{S_{i}^{n}: i \in I\right\}$ satisfy condition (I), so we have either

$$
\lim _{n \rightarrow \infty} f\left(d\left(u_{n, i}, F\right)\right) \leq \lim _{n \rightarrow \infty}\left\|x_{n}-T_{i}^{n} x_{n}\right\|=0,
$$


or

$$
\lim _{n \rightarrow \infty} f\left(d\left(x_{n}, F\right)\right) \leq \lim _{n \rightarrow \infty}\left\|x_{n}-S_{i}^{n} x_{n}\right\|=0
$$

This implies that $\lim _{n \rightarrow \infty} f\left(d\left(u_{n, i}, F\right)\right)=0$. Since $f$ is nondecreasing and $f(0)=0$, we have $\lim _{n \rightarrow \infty} d\left(u_{n, i}, F\right)=0$. The rest of the proof follows from Remark 2.2 and is, therefore, omitted.

As another application of Theorem 2.1, we establish a result for finding a common element in the set of solutions of a finite family of generalized equilibrium problems and the set of common zeros of two finite families of maximal monotone operators on a Banach space.

First we recall some preliminary concepts as follows.

A multi-valued operator $T: E \rightarrow E^{*}$ is said to be monotone if for any $x, y \in E$ and $x^{*}, y^{*} \in$ $E^{*}$ with $x^{*} \in T x$ and $y^{*} \in T y$ it holds that

$$
\left\langle x^{*}-y^{*}, x-y\right\rangle \geq 0
$$

A point $u \in E$ satisfying $0 \in T u$ is called a zero of $T$ and the set of all such points is denoted by $T^{-1} 0 . T$ is said to be maximal monotone if $T$ has no monotone extension. Equivalently, $T$ is maximal monotone if the graph of $T$, i.e., $G(T)=\{(x, y): y \in T x\}$, is not properly contained in the graph of any other monotone operator ( $c f$. [27], p.264).

Let $E$ be a strictly convex, reflexive and smooth Banach space and let $T: E \rightarrow E^{*}$ be a maximal monotone operator. For a positive real number $r$, we can define a single-valued mapping $J_{r}: E \rightarrow D(T)$ by $J_{r}(x)=(J+r T)^{-1} J x$ for each $x \in E$. This mapping is called resolvent of $T$ for $r>0$. It is known that if $T$ is maximal monotone, then $T^{-1} 0=F\left(J_{r}\right)$ for each $r>0$. Moreover, $T^{-1} 0$ is a closed convex subset of $E$. For each $r>0$, we can define Yosida approximation of $T$ by $Y_{r} x=\frac{1}{r}\left(J x-J_{r} x\right)$ for all $x \in E$. We know that $Y_{r} x \in T\left(J_{r} x\right)$; for more details, see [9].

Theorem 2.4 Let $C$ be a nonempty closed convex subset of a strictly convex, reflexive and smooth Banach space E having the Kadec-Klee property. Let $T_{i}, S_{i}: E \rightarrow E^{*}, i=1,2,3, \ldots, N$, be two finite families of maximal monotone operators and let $J_{r, i}^{T_{i}}$ and $J_{r, i}^{S_{i}}$ be the corresponding finite families of resolvents of $T_{i}$ and $S_{i}$, respectively, where $r>0$. Let $f_{n}: C \times C \rightarrow \mathbb{R}$, $n=1,2,3, \ldots, N$, be a finite family of bifunctions satisfying (A1)-(A4). Let $\left\{\alpha_{n, i}\right\},\left\{\beta_{n, i}\right\}$ and $\left\{\gamma_{n, i}\right\}$ be three sequences in $[0,1]$ such that $\alpha_{n, i}+\beta_{n, i}+\gamma_{n, i}=1$ and $\left\{r_{n}\right\} \subset(0, \infty)$ satisfying

(C1) $\liminf _{n \rightarrow \infty} r_{n}>0$.

Assume that $F:=\left[\bigcap_{i=1}^{N} T_{i}^{-1}\right] \cap\left[\bigcap_{i=1}^{N} S_{i}^{-1}\right] \cap\left[\bigcap_{n=1}^{N} G E P\left(f_{n}\right)\right] \neq \emptyset$. Then the sequence $\left\{x_{n}\right\}$ generated by

$$
\left\{\begin{array}{l}
x_{0} \in C_{0}=C, \\
y_{n, i}=J^{-1}\left(\alpha_{n, i} J x_{n}+\beta_{n, i} J J_{r_{n, i}}^{T_{i}} x_{n}+\gamma_{n, i} J J_{r_{n, i}}^{S_{i}} x_{n}\right), \\
f_{n}\left(u_{n, i}, y\right)+\left\langle A_{n} u_{n, i}, y-u_{n, i}\right\rangle+\frac{1}{r_{n}}\left\langle y-u_{n, i}, J u_{n, i}-J y_{n, i}\right\rangle \geq 0 \quad \text { for all } y \in C, \\
C_{n+1}=\left\{z \in C_{n}: \sup _{i \geq 1} \varphi\left(z, u_{n, i}\right) \leq \varphi\left(z, x_{n}\right)+\Theta_{n}\right\}, \\
x_{n+1}=\Pi_{C_{n+1}} x_{0}, \quad n \geq 0
\end{array}\right.
$$

converges strongly to $\Pi_{F} x_{0}$, where $\Pi_{F}$ is the generalized projection of $E$ onto $F$ and $\Theta_{n}:=$ $\left(1-\alpha_{n, i}\right)\left\{k_{n} \sup _{u \in F} \xi\left(\varphi\left(u, x_{n}\right)\right)+\eta_{n}\right\}$. 
Proof The first part of this proof - $J_{r_{n, i}}^{T_{i}}$ (resp. $J_{r_{n, i}}^{S_{i}}$ ) are relatively nonexpansive mappings for each $i \geq 1$ - is essentially due to Matsushita and Takahashi (cf. [27, p.265]) which we include for completeness. Note that $\widehat{F}\left(J_{r_{n, i}}^{T_{i}}\right) \subset T_{i}^{-1} 0$ for each $i=1,2,3, \ldots, N$. Let $p \in$ $\widehat{F}\left(J_{r_{n, i}}^{T_{i}}\right)$. Then there exists $\left\{z_{n}\right\} \subset E$ such that $z_{n} \rightarrow p$ and $\lim _{n \rightarrow \infty}\left\|z_{n}-J_{r_{n, i}}^{T_{i}} z_{n}\right\|=0$. Since $\liminf _{n \rightarrow \infty} r_{n}>0$, we have

$$
\frac{1}{r_{n, i}}\left\|z_{n}-J J_{r_{n, i}}^{T_{i}} z_{n}\right\| \rightarrow 0
$$

It follows from $\frac{1}{r_{n, i}}\left(J z_{n}-J J r_{n, i}^{T_{i}} z_{n}\right) \in T_{i}\left(J_{r_{n}}^{T_{i}} z_{n}\right)$ and the monotonicity of $T_{i}$ that

$$
\left\langle w-J_{r_{n, i}}^{T_{i}} z_{n}, w^{*}-\frac{1}{r_{n}}\left(J z_{n}-J J_{r_{n, i}}^{T_{i}} z_{n}\right)\right\rangle \geq 0
$$

for all $w \in D\left(T_{i}\right)$ and $w^{*} \in T_{i} w$. Letting $n \rightarrow \infty$, we have

$$
\left\langle w-p, w^{*}\right\rangle \geq 0
$$

for all $w \in D\left(T_{i}\right)$ and $w^{*} \in T_{i} w$. Therefore, from the maximality of $T_{i}$, we obtain that $p \in$ $T_{i}^{-1} 0$. On the other hand, $T_{i}^{-1} 0=F\left(J_{r, i}^{T_{i}}\right)$ and $F\left(J_{r, i}^{T_{i}}\right) \subset \widehat{F}\left(J_{r, i}^{T_{i}}\right)$, so we have $T_{i}^{-1} 0=F\left(J_{r, i}^{T_{i}}\right)=$ $\widehat{F}\left(J_{r, i}^{T_{i}}\right)$ for each $i=1,2,3, \ldots, N$. Similarly, we can show that $p \in S_{i}^{-1} 0$ and $S_{i}^{-1} 0=F\left(\left(_{r, i}^{S_{i}}\right)=\right.$ $\widehat{F}\left(J_{r, i}^{S_{i}}\right)$ for each $i=1,2,3, \ldots, N$. Moreover, the resolvent $J_{r, i}^{T_{i}}$ of $T_{i}$ (resp. $J_{r, i}^{S_{i}}$ of $S_{i}$ ) with $r>0$ satisfies $\varphi\left(u, J_{r, i}^{T_{i}} x\right) \leq \varphi(u, x)$ for all $u \in F\left(J_{r, i}^{T_{i}}\right)$ and $x \in E\left(\operatorname{resp} . \varphi\left(u, J_{r, i}^{S_{i}} x\right) \leq \varphi(u, x)\right.$ for all $u \in$ $F\left(J_{r, i}^{S_{i}}\right)$ and $x \in E$ ). Hence $J_{r, i}^{T_{i}}$ and $J_{r, i}^{S_{i}}$ are relatively nonexpansive mappings. Since the class of relatively nonexpansive mappings is properly contained in the class of total quasi- $\varphi$ asymptotically nonexpansive mappings, for a finitely many mappings case, one can derive the desired result from Theorem 2.1.

Remark 2.5 It is worth to mention that Theorem 2.1 and Theorem 2.4 improve and generalize various results available in the current literature. In particular, we highlight some significant features of both these theorems as follows.

(i) Algorithm (2.1) is comparatively more general and computationally simpler than the algorithms which appeared in $[12,18]$ and [22], respectively, in the context of (two) countable families of total quasi- $\varphi$-asymptotically nonexpansive mappings.

(ii) Theorem 2.1 improves and extends [12, Theorem 3.1], [16], [21, Theorem 3.1] and [22, Theorem 3.1] for two countable families of total quasi- $\varphi$-asymptotically nonexpansive mappings and a finite family of generalized equilibrium problems.

(iii) Theorem 2.4 improves and extends [12, Theorem 4.1] for two finite families of maximal monotone operators. Moreover, our algorithm (2.14) is computationally simpler in comparison with algorithm (4.3) in [12, Theorem 3.1].

(iv) Theorem 2.4 improves and extends [28, Theorem 4.1] for two finite families of maximal monotone operators in the more general domain of a strictly convex, reflexive and smooth Banach space $E$.

(v) In the context of an approximate common solution for an equilibrium problem, a maximal monotone operator and a countable family of relatively nonexpansive mappings, algorithms (2.1) and (2.14) are more general and computationally simpler in comparison with algorithms (3.1) and (4.2), respectively, in [28]. 
(vi) Algorithm (2.14) is computationally simpler in comparison with algorithms (3.6) in [29] and (3.1) in [30]. Moreover, Theorem 2.4 improves [29, Theorem 3.2] and [30, Theorem 3.1] by removing the $H_{n}$ and $W_{n}$ conditions in the corresponding algorithms.

(vii) Theorem 2.4 is an analogue of [17, Theorem 3.1] for the approximation of a common zero for a finite family of maximal monotone operators.

\section{Competing interests}

The authors declare that they have no competing interests.

\section{Authors' contributions}

All authors contributed equally and significantly in writing this paper. All authors read and approved the final manuscript.

\section{Author details}

${ }^{1}$ Department of Mathematics, The Islamia University of Bahawalpur, Bahawalpur, 63100, Pakistan. ${ }^{2}$ Department of Mathematics and Statistics, King Fahd University of Petroleum and Minerals, Dhahran, 3126, Saudi Arabia.

\section{Acknowledgements}

We are very grateful to anonymous referees for their critical and useful comments. The author MAA Khan gratefully acknowledges the support of Higher Education Commission (HEC) of Pakistan. The authors H Fukhar-ud-din and AR Khan are grateful to King Fahd University of Petroleum and Minerals for supporting the research project IN121037.

Received: 24 September 2013 Accepted: 19 February 2014 Published: 06 Mar 2014

\section{References}

1. Moudafi, A: Second order differential proximal methods for equilibrium problems. J. Inequal. Pure Appl. Math. 4, 18 (2003)

2. Blum, E, Oettli, W: From optimization and variational inequalities to equilibrium problems. Math. Stud. 63, 123-145 (1994)

3. Khan, MAA, Fukhar-ud-din, H: Strong convergence by the shrinking effect of two half-spaces. Fixed Point Theory Appl. 2013, Article ID 30 (2013). doi:10.1186/1687-1812-2013-30

4. Moudafi, A, Thera, M: Proximal and Dynamical Approaches to Equilibrium Problems. Lecture Notes in Economics and Mathematical Systems, vol. 477, pp. 187-201. Springer, Berlin (1999)

5. Alber, Y: Metric and generalized projection operators in Banach spaces: properties and applications. In: Kartsatos, AG (ed.) Theory and Applications of Nonlinear Operators of Accretive and Monotone Type, pp. 15-50. Dekker, New York (1996)

6. Cho, YJ, Zhou, H, Guo, G: Weak and strong convergence theorems for three-step iterations with errors for asymptotically nonexpansive mappings. Comput. Math. Appl. 47, 707-717 (2004)

7. Ibraki, T, Kimura, Y, Takahashi, W: Convergence theorems for generalized projections and maximal monotone operators in a Banach space. Abstr. Appl. Anal. 2003, 621-629 (2003)

8. Barbu, V: Nonlinear Differential Equations of Monotone Types in Banach Spaces. Springer Monographs in Mathematics. Springer, Berlin (2010)

9. Takahashi, W: Nonlinear Functional Analysis. Yokohama Publishers, Yokohama (2000)

10. Cioranescu, I: Geometry of Banach spaces. In: Duality Mappings and Nonlinear Problems. Kluwer, Dordrecht (1990)

11. Reich, S: A weak convergence theorem for the alternating method with Bregman distances. In: Theory and Applications of Nonlinear Operators of Accretive and Monotone Type. Lect. Notes Pure Appl. Math., vol. 178, pp. 313-318. Dekker, New York (1996)

12. Chang, SS, Joseph Lee, HW, Chan, CK: A new hybrid method for solving generalized equilibrium problem variational inequality and obtaining common fixed point in Banach spaces with applications. Nonlinear Anal. 73, 2260-2270 (2010)

13. Cholamjiak, P, Suantai, S: Convergence analysis for a system of equilibrium problems and a countable family of relatively quasi-nonexpansive mappings in Banach spaces. Abstr. Appl. Anal. 2010, Article ID 141376 (2010)

14. Fukhar-ud-din, H, Khan, MAA: A new implicit hybrid algorithm for an equilibrium problem and a countable family of relatively nonexpansive mappings in Banach spaces. Thai J. Math. 10(1), 13-23 (2012)

15. Li, HY, Su, YF: Strong convergence theorems by a new hybrid for equilibrium problems and variational inequality problems. Nonlinear Anal. 72, 847-855 (2010)

16. Zuo, P, Chang, SS, Liu, M: On a hybrid algorithm for a finite family of total quasi- $\varphi$-asymptotically nonexpansive mapping in Banach spaces. Fixed Point Theory Appl. 2012, Article ID 70 (2012). doi:10.1186/1687-1812-2012-70

17. Nammanee, K, Suantai, S, Cholamjiak, P: Convergence theorems for maximal monotone operators, relatively nonexpansive mappings and equilibrium problems. J. Appl. Math. 2012, Article ID 16 (2012). doi:10.1155/2012/804538

18. Qin, X, Cho, SY, Kang, SM: On hybrid projection methods for asymptotically quasi- $\varphi$-nonexpansive mappings. Appl. Math. Comput. 215(11), 3874-3883 (2010)

19. Saewan, S, Kumam, P: Convergence theorems for uniformly quasi-asymptotically nonexpansive mappings, generalized equilibrium problems and variational inequalities. J. Inequal. Appl. 2011, Article ID 96 (2011). doi:10.1186/1029-242X-2011-96 
20. Saewan, S, Kumam, P: The shrinking projection method for solving generalized equilibrium problem and common fixed points for asymptotically quasi- $\varphi$-nonexpansive mappings. Fixed Point Theory Appl. 2011, Article ID 9 (2011). doi:10.1186/1687-1812-2011-9

21. Takahashi, W, Zembayashi, K: Strong convergence theorem by a new hybrid method for equilibrium problems and relatively nonexpansive mappings. Fixed Point Theory Appl. 2008, Article ID 528476 (2008)

22. Wattanawitoon, K, Kumam, P: Strong convergence theorems by a new hybrid projection algorithm for fixed point problem and equilibrium problems of two relatively quasi-nonexpansive mappings. Nonlinear Anal. Hybrid Syst. 3, 11-20 (2009)

23. Kimura, Y, Takahashi, W: On a hybrid method for a family of relatively nonexpansive mappings in a Banach space. J. Math. Anal. Appl. 357, 356-363 (2009)

24. Beer, G: Topologies on Closed and Convex Sets. Kluwer Academic, Dordrecht (1993)

25. Kamimura, S, Takahashi, W: Strong convergence of a proximal-type algorithm in a Banach space. SIAM J. Optim. 13, 938-945 (2002)

26. Combettes, PL, Hirstoaga, SA: Equilibrium programming in Hilbert spaces. J. Nonlinear Convex Anal. 6, 117-136 (2005)

27. Matsushita, S, Takahashi, W: A strong convergence theorem for relatively nonexpansive mappings in a Banach space. J. Approx. Theory 134, 257-266 (2005)

28. Ceng, LC, Guu, SM, Hu, HY, Yao, JC: Hybrid shrinking projection method for a generalized equilibrium problem, a maximal monotone operator and a countable family of relatively nonexpansive mappings. Comput. Math. Appl. 61, 2468-2479 (2011)

29. Zeng, LC, Lin, YC, Yao, JC: Iterative schemes for generalized equilibrium problem and two maximal monotone operators. Fixed Point Theory Appl. 2009, Article ID 896252 (2009)

30. Zeng, LC, Ansari, QH, Shyu, DS, Yao, JC: Strong and weak convergence theorems for equilibrium problems and zeros of maximal monotone operators. Fixed Point Theory Appl. 2010, Article ID 590278 (2010)

10.1186/1687-1812-2014-59

Cite this article as: Khan et al:: Mosco convergence results for common fixed point problems and generalized equilibrium problems in Banach spaces. Fixed Point Theory and Applications 2014, 2014:59

\section{Submit your manuscript to a SpringerOpen ${ }^{\circ}$ journal and benefit from:}

- Convenient online submission

- Rigorous peer review

- Immediate publication on acceptance

Open access: articles freely available online

- High visibility within the field

- Retaining the copyright to your article 